\title{
An Incremental Topology Control Algorithm for Wireless Mesh Networks*
}

\author{
Mani Malekesmaeili ${ }^{1, * *}$, Mehdi Soltan $^{2}$, and Mohsen Shiva ${ }^{3, * * *}$ \\ ${ }^{1,3}$ School of Electrical and Computer Engineering, University of Tehran, Tehran 14399, Iran \\ ${ }^{2}$ STAR Lab., Stanford University, Stanford, CA, USA \\ m.malekesmaeeli@ece.ut.ac.ir, msoltan@wireless.stanford.edu, \\ mshiva@ut.ac.ir
}

\begin{abstract}
Wireless Mesh Networks (WMNs) with a promising future in wireless backhauling applications have attracted the attention of many network research centers and academic researches. High throughput and low delay as essential requirements of a wireless back-bone network demand new topology control algorithms as well as new optimization metrics. In this paper, a topology control algorithm compliant with the structure of WMNs is proposed, as well as a new metric combining the characteristics of different network layers. The proposed metric is compared with the conventional ones such as distance and interference where even an increase of over $100 \%$ in the capacity of the network is presented by applying this metric.
\end{abstract}

Keywords: Ad-hoc networks, collision domain, collision domain based topology control, topology control, wireless mesh networks, wireless backbone.

\section{Introduction}

Recently, Wireless Mesh Network (WMN) [1], as a promising newcomer in wireless access technology, has caught the attention of networking industries. Constructing a wireless backbone that provides access to Internet (or any database) is probably the most prominent application of WMNs. Large capacity and low delay as the two essential requirements in WMNs, as well as any backhaul network, demand new approaches for Topology Control (TC) and routing other than the ones available for ad-hoc wireless networks. In this paper, we focus on topology control in WMNs and introduce a new metric compliant with the needs of WMNs.

Considering the traffic pattern of WMNs, the existing TC algorithms do not suit WMNs in terms of throughput and fairness. The need for new algorithms, conforming to the requirements and characteristics of WMNs, is, therefore, perfectly felt. Other than the algorithms, the existing metrics such as the average link length, mean power, or even the number of interfering nodes, do not practically aim at fairness and throughput maximization. In conventional TC algorithms, for ad-hoc networks, direction of data streams on a link affects neither the metric nor the algorithm's procedure. While data

This work was supported by Iran Telecommunication Research Center (ITRC).

** Student Member IEEE.

**** Member, IEEE. 
directions are randomly distributed and are not of importance in ad-hoc networks, data streams in backbone WMNs [1] are mainly from gateways to end users. Considering this fact, directions of data streams are of great importance in WMNs; so either the existing TC algorithms should change to match the WMN applications or new TC algorithms should be developed. A change in a conventional algorithm can be a change in its metric. In this paper, we introduce a new metric called the Collision Domain (CD). In [2], CD is introduced as a tool for estimating the capacity of WMNs. It is shown that with certain modifications, CD can be used as a metric for TC to optimize the WMNs' performance. Thus, in so doing a new algorithm should also be developed.

The paper is organized as follows: in Section 2, we briefly refer to TC in ad-hoc networks. In Section 3, CD is firstly introduced (with certain changes to the original definition in [2]) and later the CD based topology control algorithm (CDTC) is presented. In Section 4, the simulation results are given, and finally the paper is concluded in Section 5.

\section{Topology Control in Ad-Hoc Networks}

The term of topology control has not been precisely defined in wireless networks; however, any algorithm that deals with the various aspects of the topology of a network, including nodes' positioning, network construction, link adoption, channel assignment, power control, antenna beam-forming, and even routing can be counted as topology control. Finally, a TC algorithm, directly or indirectly, provides the prerequisites for having a connected network.

There exists lots of works and papers on topology control for multi-hop wireless networks, and many different algorithms corresponding to their special requirements are developed. These requirements stem from the specific applications of the network, e.g. sensor networks or peer-to-peer, and vary from low power consumption and low number of hops to low congestion, and low delay. Full connectivity is a must in almost all of these algorithms, i.e. these algorithms aim at a full connected network with the minimum delay or the minimum average number of hops, etc. A great number of topology control algorithms for ad-hoc networks are developed to reduce transmission power in the network. Most of these algorithms take link lengths as a metric (as it is related to power with an exponent factor of 2 to 4) and claim they reduce interference implicitly. Local Minimum Spanning Tree [3], cone-based [4], and relay region based topology control [5] are among the known examples of these algorithms. It is pointed out, in [6] and [7], that reducing power does not necessarily decrease interference; they have attempted to enter interference directly into TC. These two papers and some others have each proposed a method for measuring the interference of a link. A basic model for measuring the interference of a link as a metric for TC is simply the number of active nodes in the transmission range of either ends of that link. In [8], a robust interference model is proposed for wireless ad-hoc networks. For more details on the models, and also the algorithm procedures one can refer to [6, 7, and 8]. While interference is a suitable metric for most ad-hoc networks, however, it does not match the properties of WMNs completely. Although there have been some researches in TC for WMNs, nonetheless they are mostly involved with either the channel assignment [9] or the directional antennas. The existing commercial wireless network interfaces support one channel per radio; so for a multiple channel network, the number of 
network interfaces should be as many as the number of the channels in a node, thus raising the cost of its implementation. Directional antenna designs have not only increasing costs, but also their dynamic pattern forming techniques have not been fully developed yet. Considering the above, a practical TC algorithm for current WMNs, should focus on the link adoption and the power control on single channel networks. To the best of our knowledge, there has not been any attempt for TC devoted to WMNs in single channel, omni-directional networks (a common case in the existing wireless multi-hop networks). This paper may be the first of its own in topology control for single-channel WMNs. In the next section, the collision domain (CD) load as a new metric that is well suited for a backbone WMN is introduced.

\section{Topology Control in WMNs}

\subsection{Collision Domain Load}

The idea of collision domain has stemmed from a paper by Jangeun et al [2]. Authors introduced the concept of collision domain for predicting the capacity of WMNs, wherein a unidirectional traffic from nodes to gateways on the network is assumed and the capacity of a WMN is estimated. In a backbone WMN, traffic is mostly from gateways to nodes (download traffic). In the following we introduce CD [2] with certain changes to have it well-matched to the characteristics of WMNs infrastructure.

$\mathrm{CD}$ of a link is an area where all other nodes or links should be silent (idle) for a successful transmission on that link. The CD load of a link is the total amount of data that is to be transmitted on that domain including the link itself. This load is simply referred to as the $\mathrm{CD}$ in the rest of the paper. The maximum available download rate can be estimated by the maximum existing collision domain load. As downloading from gateways (file downloading, web browsing, watching videos online, and etc.) is the dominant traffic load in an access network, like WMN, the maximum available download rate can be counted as the nominal capacity of a wireless network. The lower is the maximum CD, the larger will be the capacity of the network. Rightfulness of the above statement is proved in [2] by the simulations. It is shown that the network capacity can be precisely estimated using the maximum CD. For more information on assumptions and conclusions one can refer to [2]. CD not only shows the interference but also represents the load of the interfering nodes. For a WMN backhaul network, power consumption is not of much importance, thus, a minimum power TC algorithm does not suit these networks. Considering the above, it can be concluded that $\mathrm{CD}$ is superior to any of the other metrics proposed so far. It states the characteristics of a link more comprehensively by combining the Media Access Control (MAC) characteristics (like collision), with the amount of load obtained from the application layer, and routing rules (the network layer characteristics).

From the above it can be inferred that $\mathrm{CD}$ itself is dependent on the topology and the route that transfers the load to a specific node. Therefore, the existing TC algorithms cannot be employed for a topology construction with $\mathrm{CD}$ as the metric. It seems that because of CD's dependency on the TC result, there exists no direct optimal solution to the problem. Heuristic methods like the Genetic Algorithms or Ant Colony can be considered as sub-optimal solutions to this kind of "result depends on procedure" problem. For limited area low density networks, with a logically small 
number of available links, these methods are fine and will generate near optimal results; but for larger areas and a high number of links, as the number of variables grow, these methods will become almost impractical. Number of local minima or maxima of a real world problem (including TC) grows almost exponentially with the number of the variables. This causes an exponential increase in the complexity and run-time of a heuristic optimization problem along with a decrease in the optimality of its algorithm due to the increase in the probability that the algorithm gets stuck in a local extreme.

An important characteristic of a wireless mesh backbone (and also generally any adhoc network) is its self-reconfiguration, self-healing, and self-reconstruction abilities. This means that in the case that a route (e.g. a link,) or a node, (e.g. a router) fails, the network should be able to reconstruct itself and the services should not be interrupted. Therefore, a TC algorithm used in WMN should be able to be run many times, fast enough, and with the least change. This is not the case in time-consuming heuristic methods, which even for identical cases may generate totally different results.

In the next section, we introduce a new TC algorithm that uses CD as a metric. In addition to the above, the algorithm itself is devoted to WMNs. Other metrics like transmission power, interference, etc. can be used with this algorithm. The developed algorithm is sub-optimal, but runs fast enough and the result of the algorithm does not change fundamentally with small changes. The results in Section V show the superiority of $\mathrm{CD}$ to the other available metrics.

\subsection{Collision Domain Based Topology Control (CDTC)}

As pointed out in Section 2, for a TC algorithm to be practical, it should consider link adoption and power management for single channel multi-hop wireless networks. A new metric conforming to the characteristics of a wireless backhaul network based on the collision domain of links was proposed in the previous section. Here, we present a $\mathrm{TC}$ algorithm based on CD. It was mentioned before that $\mathrm{CD}$ is itself dependent on routes and topology; so, developing a topology control algorithm that minimizes the maximum collision domain load for any topography is impossible. A practical way for handling such a problem is to ignore optimality and look for the best sub-optimal practical algorithm. Our proposed algorithm is also a suboptimal one shown in Fig.1. The proposed algorithm not only determines the links to be used, but also designates the routes from any node to gateways. Therefore, other than TC it can perform routing and can eliminate the need for a routing algorithm for download traffic.

The proposed algorithm, as shown in Fig. 1, assumes an initial local-weight for non-gateway nodes. This local-weight is a nominee of the local traffic of each node; assuming all nodes request (can request) the same amount of traffic (this is fairness by some means), the weight of unity is applied to all nodes except the gateways. This weight can be refined further in an operating network using a feedback from the application layer.

For the proposed algorithm to be more compatible with specific traffic pattern protocols of higher levels, the required load can be estimated and sent to TC as its local-weight. The topology refreshes as significant changes occur in local-weights. The other optimization parameter, the transient-weight of a node, is an accumulation of the local-weight of nodes that receive their traffic through this node. This weight distinguishes $\mathrm{CD}$ from interference. 


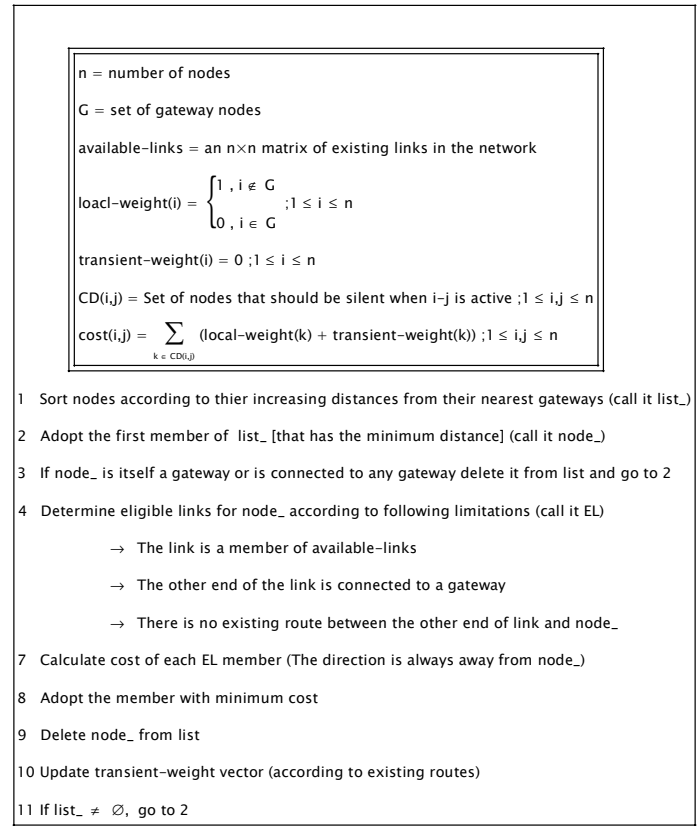

Fig. 1. Collision Domain based Topology Control (CDTC)

This algorithm is designed for WMNs and aims at a topology with the maximum available capacity. It uses CD as its optimization metric, however, any other conventional metric that is used for TC can also be used in this algorithm. For other metrics only the cost function should change. If distance is the metric, cost of a link between nodes "i" and " $\mathrm{j}$ " [ $[$ cost $(\mathrm{i}, \mathrm{j})]$ will be simply the distance between these two nodes; and the interference can be simply calculated by omitting the transient-weight factors from the formula in Fig.1. Whatever the metric, the algorithm will generate a topology that connects each router to a proper gateway. It dictates both the links and routes to be chosen for connecting a router to its gateway, but it does not generate routes for connecting any arbitrary pair of nodes. So, peer to peer traffic that has a small share in the traffic pattern of backhaul networks can be handled using the existing ad-hoc routing algorithms. As we have only considered the unidirectional download traffic from gateways to nodes, the maximum CD in the network determines the nominal capacity of the network. It is proved in [2] that under certain conditions, which are also fulfilled in our network, the nominal capacity of a WMN can be calculated as the nominal MAC layer capacity divided by the maximum $\mathrm{CD}$ in the network. Thus, minimizing the maximum $\mathrm{CD}$ will maximize the capacity. In the section that follows, the superiority of the proposed metric to distance and interference in terms of the maximum CD is presented.

\section{Simulation Results}

In this section, we compare the proposed metric (CD) is compared to two other metrics, namely interference and power (distance), using the algorithm shown in 
Fig. 1. In the simulations we have considered the download traffic. As mentioned in the previous sections, in a backbone WMN, download traffic dominates the other types of traffic such as the peer-to-peer traffic. It is this traffic pattern that differentiates WMNs from stationary ad-hoc multi-hop networks. So, for an algorithm to be suitable for WMNs this type of traffic should be considered. In [2], a method is proposed and confirmed to estimate the nominal capacity of a WMN. As our simulations match the assumptions of the said paper, we use the method proposed there to calculate the nominal capacity of our presented topologies.

Simulations are done for backbone WMNs, i.e. clients of the final hop are not considered. Since each router acts as an access point for its clients, and all clients of a specific router can only communicate with other clients using their access point, the responsibility of a backbone WMN is to deliver data packets of any client to their corresponding access point. This is why considering or not considering the last hop (from access point to client) does not affect the performance of a WMN. Fig. 2 shows a sample topology resulted from CDTC. 49 nodes (routers) are distributed distancing 120 to 200 meter from adjacent nodes in a square shaped area.

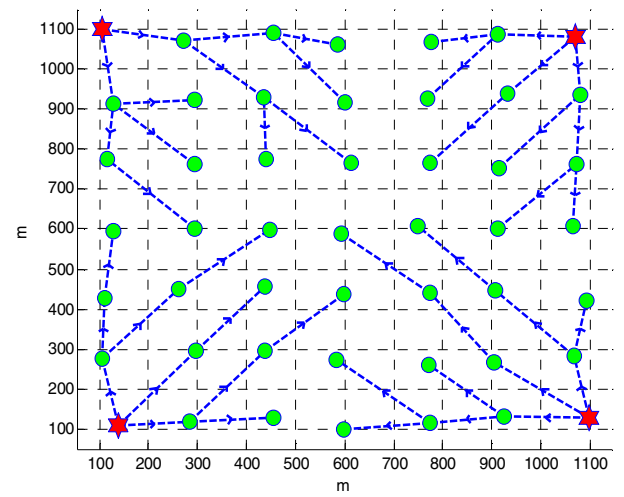

Fig. 2. CDTC result for a 40 node network with 4 gateways in the corners

The performance of the proposed metric in terms of the maximum CD is compared to the other metrics in Fig. 3. As pointed out before, for the specific conditions of our simulations, this load is a direct indicator of the nominal capacity of the network, in which the capacity will be the nominal MAC layer capacity divided by this load. So, if the maximum CD is minimized, the capacity (the maximum download rate) of the network will be maximized. The graphs in Fig. 3 show the maximum CD for different number of nodes. The area is again square shaped, and the density of the nodes in the area is constant, i.e. increasing the number of nodes equals to increasing the area that is covered by the WMN. For each number of nodes, the maximum CD is the result of the average of 10 different node position simulations (again nodes are placed randomly distancing 120 to 200 meters from their neighbors). In Fig. 3, results are shown for two different scenarios, in (a) the center node is the gateway and in (b) the 4 corner nodes are gateways. 


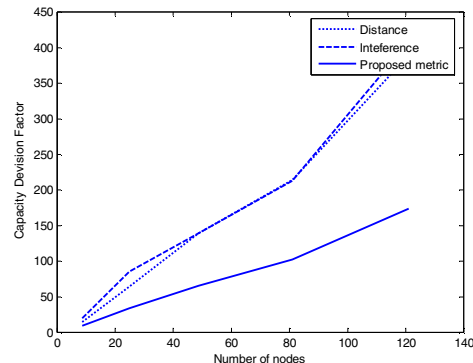

(a) 1 gateway in the center

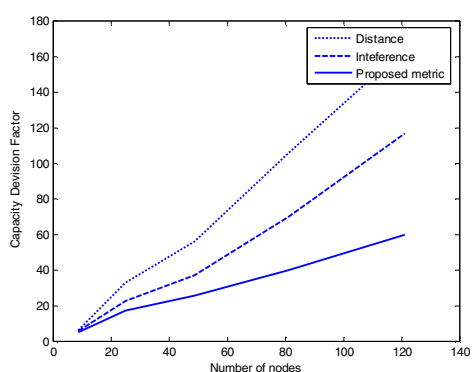

(b) 4 gateways in the corners

Fig. 3. Maximum $C D$ of the three metrics

If we assume that the nominal capacity of MAC layer is B [10], we can say that the capacity (here, the maximum download rate) is $\mathrm{B} / \max (\mathrm{CD})$ (as stated in [2]). So, we can calculate the increase in the capacity of the network (when CD is the metric as compared to when interference or distance are) simply as: (max $\left(\mathrm{CD}_{\text {other metrics}}{ }^{-}\right.$ $\left.\max \left(\mathrm{CD}_{\mathrm{CD}}\right)\right) / \max \left(\mathrm{CD}_{\mathrm{CD}}\right)$. In Fig. 4, the percentage of increase in capacity (of Fig. 3 networks) using $\mathrm{CD}$ with respect to interference and distance is shown. We can see that in both cases CD outperforms the conventional metrics, and even in some cases the capacity using $\mathrm{CD}$ is more than twice the capacity obtained from the other metrics. From the above graphs we can also conclude that in most cases interference works better than distance. The figures also highlight the efficiency of the algorithm in using $\mathrm{CD}$ as the optimization metric.

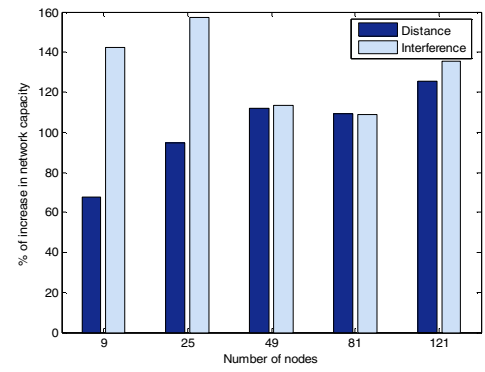

(a) 1 gateway in the center

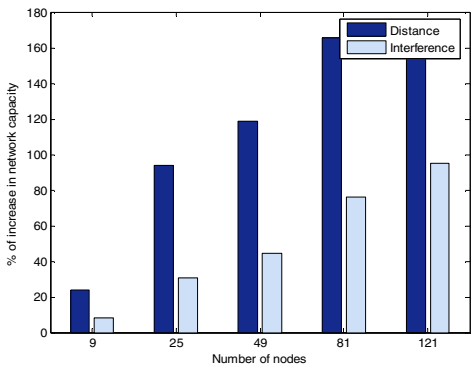

(b) 4 gateways in the corners

Fig. 4. Percentage of increase in capacity using CD compared to Interference and distance

\section{Conclusion}

In this paper, a new metric compliant with the needs of WMNs is proposed as well as an algorithm designed for download traffic in backbone WMNs. The proposed metric is tested within the algorithm and it is shown that the proposed metric outperforms the conventional metrics like distance and interference. Other than link adoption, the 
proposed metric defines the direction of data in the links, being bi-directional with the route for each node to its corresponding neighbor indicative of the possibility of its use as a routing algorithm.

\section{References}

1. I.F. Akyildiz and W. Xudong: A survey on wireless mesh networks, Communications Magazine, IEEE, Volume 43, Issue 9, (Sept. 2005) 23 - S30.

2. J. Jangeun and M.L Sichitiu: The nominal capacity of wireless mesh networks, Wireless Communications, IEEE, Volume 10, Issue 5, (Oct 2003) 8-14.

3. N. Li, J. Hou, and L. Sha: Design and Analysis of an MST-Based Topology Control Algorithm, Wireless Communications, IEEE Transactions on, Volume 4, Issue 3, (May 2005) $1195-1206$.

4. L. Li, J.Y. Halpern, P. Bahl, Y.-M. Wang, and R. Wattenhofer: A Cone-Based Distributed Topology Control Algorithm for Wireless Multi-Hop Networks, IEEE/ACM Transactions on, Volume 13, Issue 1, (Feb. 2005) $147-159$

5. V. Rodoplu, T. H. Meng: Minimum energy mobile wireless net-works, Selected Areas in Communications, IEEE Journal on, Volume 17, Issue 8, (Aug. 1999) 1333 - 1344.

6. M. Burkhart, P. v. Rickenbach, R. Wattenhofer, and A. Zollinger. : Does Topology Control Reduce Interference?, Proceedings of the 5th ACM international symposium on Mobile ad hoc networking and computing, Session: Geometry and positioning (2004) 9 - 19.

7. K. Wu, W. Liao: Interference Efficient Topology Control in Wireless Ad Hoc Networks, IEEE CCNC 2006, Volume 1, 8-10 (Jan. 2006) 411 - 415.

8. P.V. Rickenbach, S. Schmid, R. Wattenhofer, A. Zollinger: A Robust Interference Model for Wireless Ad-Hoc Networks, Parallel and Distributed Processing Symposium, 2005. Proceedings 19th IEEE International, (April 2005) 8

9. M. K. Marina, S. R. Das: A Topology Control Approach for Utilizing Multiple Channels in Multi-Radio Wireless Mesh Networks, Broadband Networks, 2005 2nd International Conference on, Volume 1, (Oct. 2005) 381 - 390.

10. J. Jun, P. Peddabachagari, and M. L. Sichitiu: Theoretical maximum throughput of IEEE 802.11 and its applications, IEEE International Symposium on Network Computing and Applications (NCA 2003) (Apr. 2003) 249-256 